\title{
LASER demarcation photocoagulation for rhegmatogenous retinal detachments
}

\begin{abstract}
Purpose To evaluate demarcation laser photocoagulation (DLP) for macula-sparing rhegmatogenous retinal detachments (RRD) with and without symptoms of posterior vitreous separation or progressive visual field defect.

Methods Retrospective, interventional, single surgeon case series of consecutive patients with RRD treated with demarcation laser photocoagulation between March 1999 and February 2008 at an academic center. The null hypothesis was that there exists no difference in the rate of progression for retinal detachment irrespective of the presence ('symptomatic') or absence ('asymptomatic') of symptoms of posterior vitreous separation or visual field defect at presentation. failure rate among eyes with symptomatic RRD.
\end{abstract}

${ }^{1}$ Medical School, Baylor College of Medicine, Houston, TX, USA

${ }^{2}$ Baylor Eye Clinic, Baylor College of Medicine, Houston, TX, USA

${ }^{3}$ Michael E DeBakey Veterans Affairs Medical Center, Houston, TX, USA

Correspondence: ER Holz, Retina \& Vitreous of Texas, 2727 Gramercy, Suite 200, Houston, TX 77025, USA Tel: +1 713 799-9975; Fax: +1 713 799-1095.

E-mail: erich@retinatexas.com

Received: 7 December 2009 Accepted in revised form: 20 July 2010

Published online: 17

September 2010

Presented at the Aegean Retina Society 2009 and at the Retina Congress 2009.
Keywords: demarcation; epiretinal membrane; LASER; photocoagulation; retinal detachment
Eye (2010) 24, 1772-1776; doi:10.1038/eye.2010.119; published online 17 September 2010
Z Al-Mohtaseb1, JL Heffez², PE Carvounis ${ }^{2,3}$ and ER Holz ${ }^{2}$

Introduction

Demarcation laser photocoagulation (DLP) refers to the placement of confluent laser photocoagulation burns (usually 3-4 rows) along the margins of a retinal detachment to completely surround it resulting in a barrier to further extension of subretinal fluid. ${ }^{1-5}$ It is less invasive than scleral buckling or vitrectomy; further, it is time-efficient, inexpensive, can be performed in the clinic, and requires no trained supporting staff, anesthesiologist or specialized operating room equipment. It is associated with minimal recovery time and, importantly, can be performed with the patient reclining at an angle or even sitting upright. Unlike pneumatic retinopexy, there are no positioning limitations post-operatively. Furthermore, complications are rare but may include epiretinal membrane (ERM) and hemorrhage. Previous series have suggested excellent results of DLP treatment for asymptomatic eyes with retinal detachments, ${ }^{2-5}$ as well as for macula-sparing persistent or recurrent retinal detachments following scleral buckling procedures, ${ }^{6}$ even with some success in delaying or avoiding vitrectomy for retinal detachments in patients with cytomegalovirus retinitis. ${ }^{7-9}$ Nonetheless, in a recent survey of retina specialists practicing in the United Kingdom and Eire, vitrectomy or buckling was favored for asymptomatic retinal detachment over observation or DLP. Furthermore, it is not established whether DLP is efficacious in eyes that have retinal detachments with visual field defect or symptoms of acute posterior hyaloid face separation (symptomatic retinal detachments). Indeed, our clinical impression has been that for symptomatic retinal detachments its efficacy is limited.

We undertook this study to further evaluate the efficacy and complication rate of DLP in eyes with symptomatic or asymptomatic primary macula-sparing rhegmatogenous 
retinal detachments (RRDs) and to better define the risk factors for failure of DLP.

\section{Materials and methods}

The clinical records of consecutive patients treated with DLP for RRDs from March 1999 till February 2008 by a single surgeon (ERH) at the Baylor Eye Clinic were retrospectively reviewed. This study was approved by our Institutional Review Board (IRB) and was compliant with the Health Insurance Portability and Accountability Act of 1996.

Included were all patients with RRDs treated with laser photocoagulation and with follow-up of at least 4 months. No patient who developed a retinal detachment before this time point was excluded. Exclusion criteria were previous intraocular surgery other than cataract extraction with intraocular lens insertion, history of Coat's disease or vitreoretinopathy predisposing to retinal detachment (eg Marfan syndrome), follow-up less than 4 months and involvement of the macula.

The patients included in the study were the patients who had elected DLP following detailed discussion of the risks, benefits of observation, surgery (vitrectomy, pneumatic retinopexy, or scleral buckle), or demarcation laser photocoagulation. Patients who elected DLP underwent same-day, in-office argon laser photocoagulation applied using either indirect ophthalmoscopy with a 25D lens or at the slitlamp with a $78 \mathrm{D}$ fundus lens and the energy and duration adjusted to produce a white reaction. Three to five rows of confluent laser photocoagulation were applied immediately posterior to the retinal detachment and anteriorly to the ora serrata. Additionally, areas of attached retina with peripheral retinal degenerations known to predispose to retinal detachment were also treated concurrently.

The patient charts were retrospectively reviewed and the following information was abstracted: symptoms (floaters, photopsiae, presence of shadows, or visual field loss), best-corrected visual acuity (BCVA), refractive error, presence or absence of Weiss ring or ERM, and lens status at presentation as well as the location, posterior and circumferential extent, and causative lesion of the RRDs. Finally, the presence or absence of a demarcation line was recorded in addition to associated findings such as lattice degeneration and previous treatment. The same information was also abstracted from the final visit.

The primary outcome was the proportion of treated eyes, which developed progression of retinal detachment beyond the laser photocoagulation scars in 'symptomatic' retinal detachments and 'asymptomatic' retinal detachments. 'Symptomatic' retinal detachments were defined as retinal detachments associated with visual field loss or symptoms of acute vitreous separation (floaters, photopsiae). 'Asymptomatic' retinal detachments were defined as retinal detachments that were found on routine examination. Secondary outcomes included the visual acuity at final visit and development of epiretinal membrane following DLP, the proportion of retinal detachments progressing in eyes with a Weiss ring or without a demarcation line at presentation or with superior location. Corrected visual acuity was measured in the clinic using Snellen charts, was converted to $\log$ MAR for statistical analysis and is presented in the manuscript as Snellen equivalent. The two-tailed Fisher-exact probability test was used to compare categorical data.

\section{Results}

A total of 27 eyes of 26 patients (average age 44 years, range 11-68) were included in the study. The characteristics of the study eyes are shown in Table 1 . The average follow-up of patients was 38 months (range: 4-96 months), and all retinae were attached anatomically at the last visit.

Of the 27 eyes, $22(81 \%)$ did not require additional treatment. Only 1 of the 14 asymptomatic patients required additional laser retinopexy (7\%) whereas 4 of 13 patients $(31 \%)$ with symptomatic macula-sparing rhegmatogenous retinal detachments required further surgery (scleral buckle and/or vitrectomy) or additional DLP $(P=0.04)$. The average BCVA of all eyes at presentation was $20 / 28$ and the average at the last visit was $20 / 27$. At final follow-up, 6 of 27 eyes had an epiretinal membrane compared with 1 of 27 at presentation $(P=0.05)$. An epiretinal membrane formed in 4 of 12 patients (33\%) with symptomatic macula-sparing RRDs compared with 1 of 14 (7\%) asymptomatic eyes $(P=0.11)$; one eye with symptomatic retinal detachment had an epiretinal membrane at presentation. Progression following DLP was not statistically more likely in eyes with a superior retinal detachment $(P=1.0)$, with flap tears $(P=0.63)$, a Weiss ring present $(P=0.32)$, without a demarcation line $(P=0.37)$, or with a retinal detachment 2 clock hours or more $(P=0.37)$.

Of the five eyes that required additional treatment, one patient had progression of posterior vitreous separation with an associated new horseshoe tear 3 weeks following DLP and was treated with repeat DLP without further progression and final BCVA 20/20. Another patient had extension of the retinal detachment through the barricade 3 days following treatment and underwent scleral buckle; BCVA at the most recent follow-up visit was $20 / 25$. An additional patient developed a posterior 
Table 1 Characteristics of study eyes at presentation

\begin{tabular}{lc}
\hline & $\mathrm{N}(\%)$ \\
\hline Gender & \\
Male & $19(73)$ \\
Female & $7(27)$ \\
Refractive error & \\
Less than 1D error & \\
1-3D myopia & $4(15)$ \\
4-10D myopia & $3(11)$ \\
>10D myopia & $7(26)$ \\
Not recorded & $4(15)$ \\
& $9(33)$ \\
Lens status & \\
Phakic & \\
Pseudophakic & $24(89)$ \\
Causative lesion & $3(11)$ \\
Horseshoe tear & \\
Atrophic hole & \\
None found & $10(37)$ \\
& $15(56)$ \\
Extent: & $2(7)$ \\
Detachment extends to equator or further & \\
posteriorly & \\
Circumferential (clock hours) & $26(96)$ \\
Superior location & \\
Inferior location & \\
Presence of demarcation line: & $2.14(\mathrm{mean})$ \\
Epiretinal membrane & $9(33)$ \\
\hline & $18(67)$ \\
& $10(37)$ \\
& $11(4)$ \\
\hline
\end{tabular}

hyaloid face separation with vitreous hemorrhage 3 months after laser treatment and had two new tears. He underwent successful vitrectomy and scleral buckle; however, his BCVA was 20/200 at the last visit. A further patient developed a new horseshoe tear 8 months after treatment, which was treated with laser retinopexy, but subretinal fluid broke through the laser and the patient underwent scleral buckling 4 months later; final BCVA was $20 / 20$. The last patient developed subretinal fluid break through the initial laser treatment and a new retinal break with associated retinal detachment 3 months after DLP, and was treated with a combined vitrectomy/scleral buckle. The patient subsequently developed a posterior subcapsular cataract and thus was $20 / 80$ at the last visit.

\section{Discussion}

Our results suggest that although there is no (or minimal) risk of progression following DLP in asymptomatic retinal detachments, there is a high failure rate following DLP for symptomatic retinal detachments.
Our results are in agreement with a previously published prospective study of asymptomatic retinal detachments extending more than two disc diameters posterior to the equator treated with DLP and followed for at least 6 months, in which only 1 in 19 (5.3\%) progressed. ${ }^{4}$ Further, in a study including 34 eyes of 31 juvenile patients with asymptomatic macula-sparing retinal detachments (RDs), most of which extended posterior to the equator three $(8.8 \%)$, required further DLP application due to progression and none $(0 \%)$ required scleral buckling or vitrectomy with an average follow-up of 3.5 years. ${ }^{5}$ Finally, our results are in agreement with those of Okun and Cibis, ${ }^{2}$ that performed DLP for dialysis-related RD anterior to the equator (presumably asymptomatic) with progression of the detachment in one of nine eyes $(11 \%)$.

Our results, however, contradict the results of a retrospective study of 34 primary and recurrent maculasparing retinal detachments that reported progression in only a single patient ( $3 \%$ ) with follow-up of at least 3 months; in this study 14 of the 34 retinal detachments had photopsiae or floaters (ie 'symptomatic'). ${ }^{3}$ The greater incidence of progression in symptomatic patients treated with DLP in our study may be explained by differences in baseline characteristics that may be related to a greater propensity to DLP failure: notably $77 \%$ of detachments in that study were anterior to the equator compared with $4 \%$ in ours, more of the eyes in our study were myopic and $18 \%$ of patients in their study had had previous retinal repair whereas all of our patients had primary RDs.

It is unclear whether DLP is superior to observation alone in patients with asymptomatic retinal detachments as the natural history of the latter is unclear. ${ }^{10-15}$ This is because of the fact that there are conflicting reports on the natural history of asymptomatic retinal detachments. ${ }^{10-15}$ Several studies suggest a very benign history with a rate of progression of about $10 \%$ or less even with several years' follow-up. ${ }^{10-12}$ Brod et al ${ }^{10}$ reported 31 eyes of 28 patients, $81 \%$ of whom had atrophic holes causing asymptomatic retinal detachments, most of which extended posterior to the equator. Only two $(6.5 \%)$ progressed during the mean 3.4 years of follow-up. ${ }^{10}$ Similarly in a prospective study of 19 eyes with 22 'subclinical' retinal detachments (extending from one disc diameter posterior to the retinal break up to two disc diameters posterior to the equator), only two (11\%) progressed during a mean of 13.4 years follow-up. ${ }^{11}$ Further, a prospective study of 18 eyes of 16 patients with retinal detachments extending posterior to the equator, all of which had demarcation lines, found no progression $(0 \%)$ to symptomatic retinal detachment during a mean follow-up of 3.8 years. ${ }^{12}$ On the other 
hand, several studies suggest a rate of progression of $40-45 \%$ in asymptomatic retinal detachments. ${ }^{13-15}$ Specifically, a retrospective study of 10 eyes with subclinical retinal detachments, followed for at least 6 months, reported that four $(40 \%)$ of these progressed. ${ }^{13}$ A further retrospective study also showed a high proportion of asymptomatic retinal detachments (9 of 20, $45 \%$ ) having progressed beyond a previous demarcation line. ${ }^{14}$ Finally, Jarrett et $a l^{15}$ published a retrospective study that showed progression in 6 of 15 retinal detachments (40\%) with a mean follow-up of 4 years. Notably, $100 \%$ of superior RDs progressed and the author noted no detriment to visual acuity as a result of observation. In summary, the natural history of asymptomatic retinal detachments is unclear rendering it impossible to gauge whether DLP is helpful or unnecessary. We believe it offers an additional measure of protection that is less expensive and may be safer than invasive surgery for patients who are uncomfortable with observation alone or who have limited access to healthcare (eg live in underserved rural areas and have difficulty accessing an ophthalmologist).

Symptomatic retinal detachments are thought to invariably progress. Demarcation laser photocoagulation prevented progression in 8 of 13 eyes in our study $(61.5 \%)$. It may be that selecting eyes with symptomatic retinal detachments of more limited extent (anterior to the equator) that are smooth and shallow, as in the study by Vrabec and Baumal, ${ }^{3}$ produces better results.

Certainly, for eyes with symptomatic retinal detachments the failure rate of $5 / 13(38.5 \%)$ is rather high in our study and higher than published rates of pneumatic retinopexy, ${ }^{16}$ scleral buckling, ${ }^{17,18}$ or pars plana vitrectomy. ${ }^{19}$ However, in patients who are apprehensive of invasive surgery or for patients who may be infirm and poor surgical candidates (eg unable to lay flat due to severe emphysema, heart failure, or severe kyphosis) or who do not have the resources for operative repair (lack of ophthalmologist trained in scleral buckling or vitrectomy) DLP may represent a good option.

In conclusion, for asymptomatic retinal detachments DLP is a relatively inexpensive, time-efficient and safe treatment. For symptomatic retinal detachments, DLP is more likely to require further surgery than scleral buckling or vitrectomy, although results may be similar when DLP is applied to detachments that are shallow and limited to anterior to the equator. For symptomatic detachments it is an option for patients who prefer a less invasive alternative, in the understanding that there is a higher likelihood for further surgery than definitive repair, or for patients who are poor surgical candidates, or those who lack resources for repair in the operating room.

\section{Summary}

What was known before

- Retina specialists prefer vitrectomy of scleral buckles over observation of demarcation.

\section{What this study adds}

- Demarcation Laser photocoagulation has a high failure rate for symptomatic retinal detachments. Demarcation laser photocoagulation is effective for asymptomatic retinal detachments.

\section{Conflict of interest}

The authors declare no conflict of interest.

\section{References}

1 Greenberg PB, Baumal CR. Laser therapy for rhegmatogenous retinal detachment. Curr Opin Ophthalmol 2001; 12: 171-174.

2 Okun E, Cibis PA. Photocoagulation in 'limited' retinal detachment and breaks without detachment. In: Alice McPherson (ed). New and Controversial Aspects of Retinal Detachment. Harper \& Row: New York, 1968; 164-171.

3 Vrabec TR, Baumal CR. Demarcation laser photocoagulation of selected macula-sparing rhegmatogenous retinal detachments. Ophthalmology 2000; 107: 1063-1067.

4 Shukla D, Maheshwari R, Kim R. Barrage laser photocoagulation for macula-sparing asymptomatic clinical rhegmatogenous retinal detachments. Eye 2007; 21: 742-745.

5 Hwang JF, Chen SN. Demarcation laser photocoagulation of macula-sparing retinal detachments in teenagers. Retina 2008; 28: 1487-1492.

6 Wong YM, Lois N. Demarcation laser therapy in the management of macular-sparing persistent subretinal fluid after scleral buckling procedures. Graefes Arch Clin Exp Ophthalmol 2006; 244: 1039-1042.

7 Davis JL, Hummer J, Feuer WJ. Laser photocoagulation for retinal detachments and retinal tears in cytomegalovirus retinitis. Ophthalmology 1997; 104: 2053-2060.

8 Vrabec TR. Laser photocoagulation repair of macula-sparing cytomegalovirus-related retinal detachment. Ophthalmology 1997; 104: 2062-2067.

9 Ahmad N, West J. Current opinion on treatment of asymptomatic retinal detachments. Eye 2007; 21: 1179-1185.

10 Brod RD, Flynn Jr HW, Lightman DA. Asymptomatic rhegmatogenous retinal detachments. Arch Ophthalmol 1995; 113: $1030-1032$.

11 Byer NE. Subclinical retinal detachment resulting from asymptomatic retinal breaks: prognosis for progression and regression. Ophthalmology 2001; 108: 1499-1503.

12 Cohen SM. Natural history of asymptomatic clinical retinal detachments. Am J Ophthalmol 2005; 139: 777-779.

13 Davis MD. Natural history of retinal breaks without detachment. Arch Ophthalmol 1974; 92: 183-194.

14 Benson WE, Nantawan P, Morse PH. Characteristics and prognosis of retinal detachments with demarcation lines. Am J Ophthalmol 1977; 84: 641-644.

15 Jarrett II WH. Retinal detachment: is reparative surgery always mandatory? Trans Am Ophthalmol Soc 1988; 86: 307-320. 
16 Tornambe PE. Pneumatic retinopexy: the evolution of case selection and surgical technique. A twelve-year study of 302 eyes. Trans Am Ophthalmol Soc 1997; 95: 551-578.

17 Kreissig I, Rose D, Jost B. Minimized surgery for retinal detachments with segmental buckling and nondrainage. An 11-year follow-up. Retina 1992; 12: 224-231.
18 Schwartz SG, Kuhl DP, McPherson AR, Holz ER, Mieler WF. Twenty-year follow-up for scleral buckling. Arch Ophthalmol 2002; 120: 325-329.

19 Johansson K, Malmsjo M, Ghosh F. Tailored vitrectomy and laser photocoagulation without scleral buckling for all primary rhegmatogenous retinal detachments. $\mathrm{Br} \mathrm{J}$ Ophthalmol 2006; 90: 1286-1291. 